\title{
smarter medicine: «Top-5-Liste» für Pädiatrie
}

Trägerschaft «smarter medicine - Choosing Wisely Switzerland» und pädiatrie schweiz

Ziel der Medizin ist es, dem Patienten eine qualitativ hochwertige Versorgung zu bieten sowie unnötige und sogar gefährliche Untersuchungen und Behandlungen zu vermeiden. Aus diesem Grund hat sich auch pädiatrie schweiz dem Trägerverein «smarter medicine Choosing Wisely Switzerland» angeschlossen. Dieser wird nicht nur von medizinischen fach- und berufsständischen Organisationen, sondern auch von Patienten- und Konsumentenorganisationen getragen und will das Bewusstsein für die Problematik der Überoder Fehlversorgung sensibilisieren.

Als Fachgesellschaft hat pädiatrie schweiz eine entsprechende «Top-5-Liste» erarbeitet mit fünf medizinischen Massnahmen, die in der Regel unnötig sind und aufgrund von Nebenwirkungen schädlich sein können. Kinder- und Allgemeinärzte und -ärztinnen sowie Patientinnen und Patienten sollten bei diesen Empfehlungen miteinander im Sinne einer shared decision making besprechen, ob nicht besser auf diese Massnahmen verzichten werden sollte, weil sie mit Risiken oder Nebenwirkungen verbundenen sind und ein Nutzen gemäss der heutigen Evidenz nicht gegeben ist. Die allgemeine Botschaft ist, dass mehr nicht immer besser ist (more is not always better).

\section{Fünf Empfehlungen}

Unsere Liste beinhaltet fünf Empfehlungen für die Behandlung von Säuglingen, Kindern und Jugendlichen, welche sowohl in der ambulanten wie auch stationären Medizin relativ häufig und gut umsetzbar sind sowie von allen Kinder- und Jugendärzten und -ärztinnen in der Schweiz in einer Umfrage als wichtig mit einem hohen Potenzial für vermeidbaren Schaden erachtet wurden.

pädiatrie schweiz ist der Ansicht, dass mit der vorhandenen Top-5-Liste ein wichtiger Schritt zur Implementierung der Initiative smarter medicine für die Kinderärzte und -ärztinnen erarbeitet wurde.

Die Schweizerische Gesellschaft für Pädiatrie empfiehlt, folgende fünf Interventionen in der Pädiatrie zu vermeiden:

\section{Die Kampagne "smarter medicine»}

Der Trägerverein «smarter medicine - Choosing Wisely Switzerland", der nebst medizinischen Fach- und Berufsorganisationen auch von Patienten- und Konsumentenorganisationen unterstützt wird, möchte die Öffentlichkeit für die Themen der Fehl- und Überversorgung sensibilisieren. Der Verein knüpft an die erfolgreiche amerikanische Initiative "Choosing Wisely" an, die zum Ziel hat, nicht nur «kluge Entscheidungen» herbeizuführen, sondern auch die offene Diskussion zwischen Ärzteschaft, Patientinnen und Patienten sowie der Öffentlichkeit zu fördern. In den nächsten Monaten werden weitere medizinische Fachgesellschaften sogenannte Top-5-Listen mit unnützen Behandlungen in ihrem Fachbereich publizieren. Die jeweiligen Empfehlungen sind auch in einer für Laien verständlichen Sprache verfügbar, um gemeinsame Entscheidungen zu unterstützen. Weitere Informationen zumTrägerverein und eine Übersicht über die bestehenden Top-5-Listen sind zu finden unter www.smartermedicine.ch.

\section{Starten Sie bei leicht bis mässig dehydrierten} Kindern nicht eine intravenöse Therapie, bevor eine orale Rehydrierung versucht worden ist. Die enterale Rehydrierung per os oder via Magensonde mit einer oralen Elektrolytlösung ist ebenso wirksam wie die intravenöse Rehydrierung und mit weniger schwerwiegenden Nebenwirkungen assoziiert. In vielen hochentwickelten Ländern kann bei leichter Dehydratation die Verwendung von verdünntem Apfelsaft oder anderen vom Kind bevorzugten Flüssigkeiten (zum Beispiel Muttermilch) eine geeignete Alternative zu Elektrolytlösungen sein. Die erfolgreiche Platzierung eines venösen Zugangs ist bei dehydrierten Kindern häufig schwierig und kann mehrere Versuche erfordern, was die Rehydrierung zusätzlich verzögert. In einigen Übersichtsarbeiten liegt die Häufigkeit des Versagens der enteralen Rehydrierung bei Kindern mit Durchfall und Erbrechen bei 5\%, bei alleinigem Durchfall sogar niedriger.

\section{Behandeln Sie eine akute Otitis media bei Kindern nicht routinemässig mit einem Antibiotikum.}

Vermeiden Sie den routinemässigen Einsatz von Antibiotika bei unkomplizierter akuter Otitis media (Mit- 


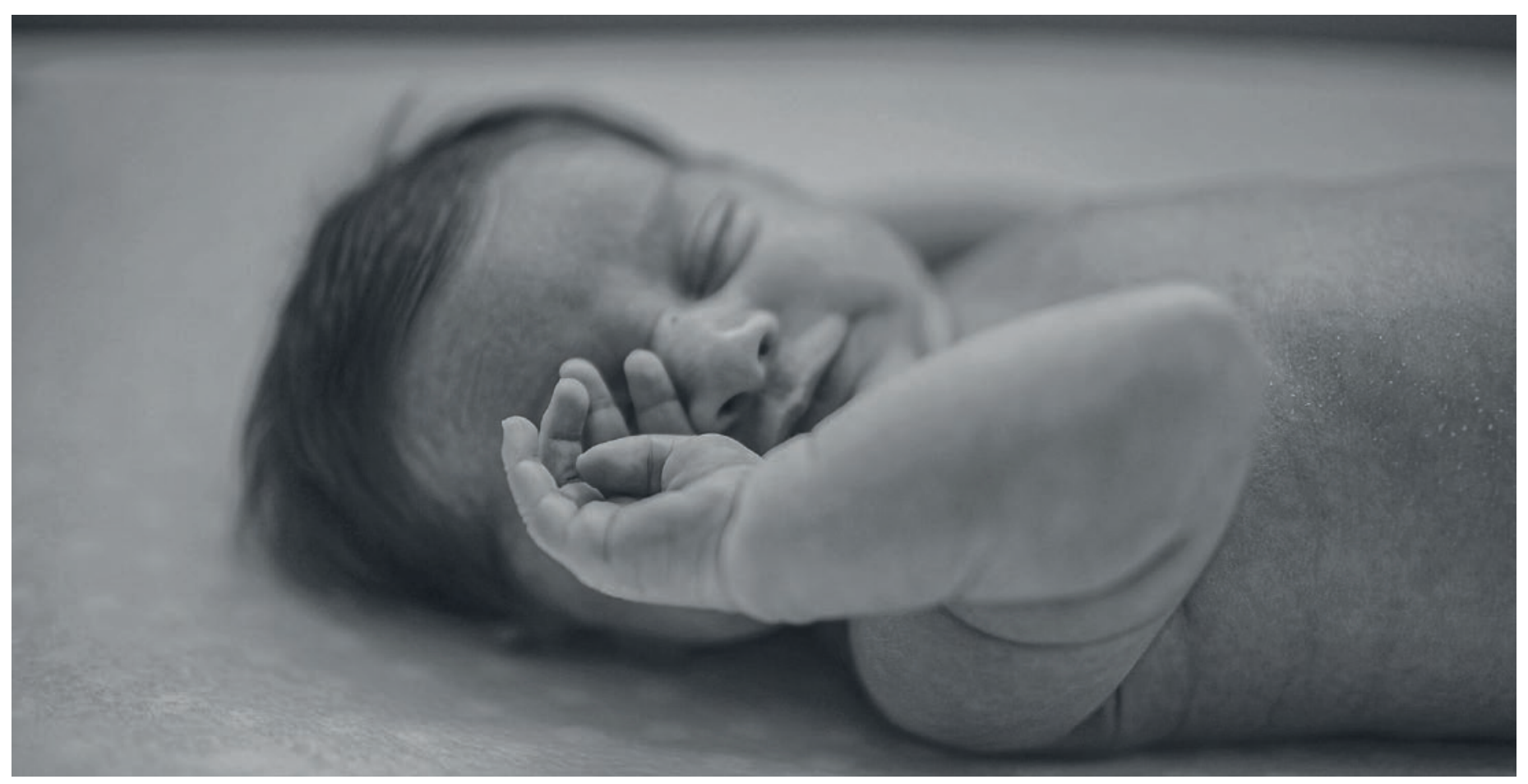

telohrentzündung) bei Kindern über 6 Monaten, da eine akute Otitis media in der Regel die Folge einer viralen Infektion der oberen Atemwege ist. Eine klinische Neubeurteilung nach 24 bis 48 Stunden unter adäquater analgetischer Therapie ist empfohlen. In den meisten Fällen kommt es zu einer spontanen Besserung der Symptome und schwere Komplikationen sind selten. Der Einsatz von Antibiotika kann resistente Bakterien fördern und Nebenwirkungen verursachen, verhindert schwere Komplikationen aber nicht.

\section{Verwenden Sie keine Hustenmedikamente bei Kindern.}

Husten ist im Allgemeinen ein normaler Abwehrmechanismus des Körpers. Es wurde gezeigt, dass sowohl chemische wie pflanzliche Hustenmedikamente gegen Erkältungen nicht wirksam sind und potenziell schwerwiegende Nebenwirkungen haben können. Viele Produkte enthalten mehr als einen Inhaltsstoff, was das Risiko einer Überdosierung erhöht, vor allem wenn sie mit anderen Medikamenten kombiniert werden.

\section{Verwenden Sie bei Säuglingen mit Bronchio- litis nicht routinemässig Steroide oder Bronchodilatatoren.}

Systemische oder inhalative Steroide haben weder einen positiven Effekt auf die Hospitalisationsrate noch auf die Dauer des Krankenhausaufenthalts bei Säuglingen mit Bronchiolitis.
Zudem zeigt die Evidenz, dass Bronchodilatatoren wie Salbutamol bei Säuglingen mit Bronchiolitis weder die Sauerstoffsättigung verbessern, die Krankenhauseinweisungen oder die Dauer des Krankenhausaufenthalts reduzieren noch die Zeit bis zum Abklingen der Erkrankung verkürzen. Salbutamol kann unerwünschte Nebenwirkungen wie Tachykardie, Verschlechterung der Sauerstoffsättigung und Tremor verursachen.

\section{Verwenden Sie Säureblocker nicht routine- mässig zur Behandlung des gastroösophagealen Reflux bei Säuglingen.}

Gastroösophagealer Reflux (GÖR) ist ein physiologischer Prozess und erfordert bei Säuglingen keine Behandlung mit Säureblockern. Die Säuresuppression verbessert weder unspezifische Symptome wie exzessives Schreien noch Aufstossen/Spucken. Der Einsatz von Säureblockern wie Protonenpumpeninhibitoren (PPI) und H2-Rezeptorantagonisten kann zu Nebenwirkungen wie häufigeren Infektionen der unteren Atemwege, Veränderungen der Darmflora oder verzögerter Magenentleerung führen und ist mit einer verminderten Knochenmineralisierung verbunden.

Wenn der Rückfluss von Mageninhalt den Alltag beeinträchtigende Symptome verursacht oder zu Komplikationen führt, liegt eine gastroösophageale Refluxkrankheit (GERD) vor. Ein Therapieversuch mit PPI sollte bei Säuglingen nicht als diagnostischer Test für GERD verwendet werden. 
Weiterführende Informationen: Eine ausführliche Literaturliste ist unter www.smartermedicine.ch verfügbar.

\section{Zur Entstehung dieser Liste}

Im November 2018 wurde die Arbeitsgruppe (AG) Choosing Wisely (CW) von pädiatrie schweiz (Schweizerische Gesellschaft für Pädiatrie) aus Vertretern der stationären und ambulanten Kinder- und Jugendmedizin gegründet. Die AG hatte in einem ersten Schritt die bereits vorhandenen pädiatrischen CW-Listen aus anderen Ländern zusammengetragen, beurteilt, redigiert, ergänzt und daraus eine erste Liste mit 65 Items erstellt. Diese wurden hinsichtlich ihrer Evidenz sowie ihrer Anwendbarkeit oder Relevanz in der Schweiz geprüft.

In einem nächsten Schritt erfolgte eine erste DelphiUmfrage mittels SurveyMonkey betreffend der Häufigkeit, Relevanz und Anwendbarkeit innerhalb der AG sowie erweitert bei Vertretern der verschiedensten Interessensgruppen und pädiatrischen Spezialisten. Dabei wurden jeweils zu den drei Fragen eine 7-PunkteLikert-Skala angewandt. Zusätzlich bestand die Option, neue Items hinzuzufügen. Basierend auf den Antworten wurde die Liste statistisch ausgewertet und hinsichtlich der Häufigkeit überarbeitet (Mean value, Standard deviation, Interquartile Range, Median), woraus eine zweite Liste mit 42 Items resultierte.

In der Folge wurden die Mitglieder von pädiatrie schweiz (ordentliche Mitglieder, Assistenten Mitglieder) in einer zweiten Delphi-Umfrage angeschrieben und aufgefordert, sich mittels der 7-Punkte-LikertSkala zur Erfassung der Relevanz zu den einzelnen
Items zu äussern. Ziel war dabei, die Top-5-Liste von Beginn an möglichst breit bei den Kinderärzten abzustützen. Zudem wurde erfragt, ob die Formulierung klar und verständlich war. Zu jedem Item konnte ein Kommentar abgegeben werden. Nach statistischer Auswertung der Antworten sowie der Kommentare wurde eine dritte Liste mit 19 Items erstellt.

Für die finale Top-5-Liste wurden schliesslich durch die AG vier Items aus der dritten und ein Item aus der zweiten Liste ausgewählt. Letzteres auch deswegen, weil dieses Item für die AG eine hohe praktische Relevanz hat und sehr gut zur smarter medicine Kampagne passt. Darüber hinaus sind auch Aspekte zur Umsetzung und Überprüfbarkeit der Massnahmen in die Erstellung der Top-5-Liste eingeflossen. Diese erste schweizerische pädiatrische Top-5-Liste betrifft nun sowohl die Praxis- wie die Spitalpädiatrie und beinhaltet vor allem therapeutische Aspekte im Sinne von do not harm.

Die finalisierte Liste wurde durch den Vorstand von pädiatrie schweiz überprüft. Nach Diskussionen und Rückmeldungen aus dem Vorstand nahm die AG eine Neubeurteilung des Items zur Bronchiolitis vor und ersetzte das ursprünglich nur knapp gewählte Item «Diagnostik bei Bronchiolitis» einstimmig durch das Item «Therapie bei Bronchiolitis». Diese nun finalisierte Top-5-Liste enthält Empfehlungen, deren Anwendung im Einzelfall zu überprüfen ist.

Bildnachweis

Jenna Norman / Unsplash

\section{Literatur}

Eine umfassende Bibliografie ist zu finden unter

www.smartermedicine.ch
Trägerverein

smartermedicine

c/o SGAIM

Monbijoustrasse 43

CH-3001 Bern

smartermedicine[at]

sgaim.ch

\author{
Arbeitsgruppe Choosing Wisely \\ pädiatrie schweiz \\ Dr. med. Corinne Wyder \\ Dr. med. Dominique Gut \\ Prof. Dr. med. Gian Paolo Ramelli \\ Prof. Dr. med. Roland Ammann \\ Dr. med. Gérald Berthet \\ Dr. sc. Ermindo Di Paolo, PhD \\ Prof. Dr. med. Mario Gheri \\ Dr. med. Benedikt Huber \\ Dr. med. Sara Schnyder \\ Dr. med. Marc Sidler
}

\section{Das Wichtigste in Kürze}

- Die Fachgesellschaft pädiatrie schweiz hat im Rahmen der Kampagne "smarter medicine» fünf Massnahmen definiert, auf die Kinder- und Jugendärzte in Absprache mit ihren $\mathrm{Pa}$ tientinnen und Patienten in der Regel verzichten können.

- Die fünf Interventionen, darunter etwa der routinemässige Einsatz von Antibiotika bei unkomplizierter akuter Otitis media, nützen den Patientinnen und Patienten nicht, haben aber unerwünschte Nebenwirkungen.

- Die Empfehlungen sind auch in einer für Laien verständlichen Sprache verfügbar, damit der Dialog zwischen Ärzteschaft, Patientinnen und Patienten sowie der Öffentlichkeit gefördert wird. 